\title{
The greatest common divisors of generalized Fibonacci and generalized Pell numbers
}

\author{
Boonyen Thongkam $^{1}$ and Nutcha Sailadda ${ }^{2}$ \\ ${ }^{1}$ Department of Mathematics, Faculty of Science \\ Ubon Ratchathani Rajabhat University \\ Ubon Ratchathani, 34000, Thailand \\ e-mail: boonyen.teubru.ac.th \\ 2 Department of Mathematics, Faculty of Education \\ Ubon Ratchathani Rajabhat University \\ Ubon Ratchathani, 34000, Thailand \\ e-mail: nutchasailadda@gmail.com
}

Received: 22 September 2017

Accepted: 31 January 2018

\begin{abstract}
Abd-Elhameed and Zeyada have introduced the generalized sequence of numbers $\left(U_{n}^{a, b, r}\right)_{n \geq 0}$ such that sequence generalizes both generalized Fibonacci numbers $\left(G_{n}^{a, b}\right)_{n \geq 0}$ and generalized Pell numbers $\left(P_{n}^{a, b}\right)_{n \geq 0}$. In the present paper, we show a study of the greatest common divisors of some $G_{n}^{a, b}, P_{n}^{a, b}$ and $U_{n}^{a, b, r}$.
\end{abstract}

Keywords: Greatest common divisor, Generalized Fibonacci number, Generalized Pell number. 2010 Mathematics Subject Classification: 11B39, 11A05.

\section{Introduction}

Let $\left(F_{n}\right)_{n \geq 0}$ be the Fibonacci sequence given by $F_{0}=0, F_{1}=1, F_{n+2}=F_{n+1}+F_{n}$ for $n \geq 0$ and let $\left(L_{n}\right)_{n \geq 0}$ be the Lucas sequence given by $L_{0}=2, L_{1}=1, L_{n+2}=L_{n+1}+L_{n}$ for $n \geq 0$. Fibonacci numbers and Lucas numbers are famous for their special and amazing properties. An important identity of Fibonacci and Lucas numbers that they mutually have is $L_{n}=F_{n-1}+F_{n+1}$ for any $n \geq 1$. Let $G_{n}$ be the generalized Fibonacci numbers given by $G_{0}^{a, b}=b-a, G_{1}^{a, b}=a$ and $G_{n+2}^{a, b}=G_{n+1}^{a, b}+G_{n}^{a, b}$ for $n \geq 0$. It is clear that such the generalized Fibonacci numbers are the generalization of both Fibonacci and Lucas numbers. In fact, we have $F_{n}=G_{n}^{1,1}$ and $L_{n}=G_{n}^{1,3}$. Koshy [5] has already proved that $G_{n+2}^{a, b}=a F_{n}+b F_{n+1}$. 
Let $\left(P_{n}\right)_{n \geq 0}$ be the Pell sequence given by $P_{0}=0, P_{1}=1, P_{n+2}=2 P_{n+1}+P_{n}$ for $n \geq 0$ and let $\left(Q_{n}\right)_{n \geq 0}$ be the Pell-Lucas sequence given by $Q_{0}=2, Q_{1}=2, Q_{n+2}=2 Q_{n+1}+Q_{n}$ for $n \geq 0$. An important identity of Pell and Pell-Lucas numbers is $Q_{n}=P_{n-1}+P_{n+1}$ for any $n \geq 1$. Abd-Elhameed and Zeyada [1] have introduced the generalized Pell numbers as $P_{0}^{a, b}=$ $b-2 a, P_{1}^{a, b}=a$ and $P_{n+2}^{a, b}=2 P_{n+1}^{a, b}+P_{n}^{a, b}$ for $n \geq 0$. It is clear that such the generalized Pell numbers are the generalization of both Pell and Pell-Lucas numbers. In fact, we have $P_{n}=P_{n}^{1,2}$ and $Q_{n}=P_{n}^{2,6}$. Furthermore, the authors [1] have introduced the new sequence of generalized numbers $\left(U_{n}^{a, b, r}\right)_{n \geq 0}$ as

$$
U_{n+2}^{a, b, r}=r U_{n+1}^{a, b, r}+U_{n}^{a, b, r} \text { where } U_{0}^{a, b, r}=b-r a \text { and } U_{1}^{a, b, r}=a .
$$

We have $G_{n}^{a, b}=U_{n}^{a, b, 1}$ and $P_{n}^{a, b}=U_{n}^{a, b, 2}$. Thus, we have the two sequences $\left(G_{n}^{a, b}\right)_{n \geq 0}$ and $\left(P_{n}^{a, b}\right)_{n \geq 0}$ are particular sequences of the more general sequence $\left(U_{n}^{a, b, r}\right)_{n \geq 0}$.

The greatest common divisor of positive integers $a$ and $b$ is the largest positive integer $d$ such that $a$ and $b$ are both multiples of $d$. Let $\operatorname{gcd}(a, b)$ represent the greatest common divisor of $a$ and $b$. The greatest common divisors of Fibonacci, Lucas, Pell and generalized Fibonacci numbers have been studied widely, which can be seen in $[2,3,4,5,6]$. It is well known that $\operatorname{gcd}\left(F_{m}, F_{n}\right)=F_{\operatorname{gcd}(m, n)}$, and $\operatorname{gcd}\left(P_{m}, P_{n}\right)=P_{\operatorname{gcd}(m, n)}$.

In this paper, we show our study of the greatest common divisors of $G_{n}^{a, b}, P_{n}^{a, b}$ and $U_{n}^{a, b, r}$.

\section{The main results}

The following theorem is well known and can be found many textbook on number theory.

Theorem 1. [7] For every non-zero integers $a$ and $b$, then $\operatorname{gcd}(a, b)=\operatorname{gcd}(a+b x, b)$ for any integer $x$.

Theorem 2. [4, 5] For every positive integers $m, n$, we have

(1) $n \mid m$ if and only if $F_{n} \mid F_{m}$,

(2) $n \mid m$ if and only if $P_{n} \mid P_{m}$.

Theorem 3. [4, 5] For every positive integers $m, n$, we have $\operatorname{gcd}\left(F_{m}, F_{n}\right)=F_{\operatorname{gcd}(m, n)}$, and $\operatorname{gcd}\left(P_{m}, P_{n}\right)=P_{\operatorname{gcd}(m, n)}$. This implies that $\operatorname{gcd}\left(F_{n}, F_{n+1}\right)=\left(P_{n}, P_{n+1}\right)=1$.

Theorem 4. For every non-zero integers $a, b, r$ and non-negative integer $n$, we have

$$
\operatorname{gcd}\left(U_{n}^{a, b, r}, U_{n+1}^{a, b, r}\right)=\operatorname{gcd}(a, b) .
$$

Proof. We will proceed by induction on $n$. It is clear in case $n=0$. Now, assume that $\operatorname{gcd}\left(U_{n}^{a, b, r}, U_{n+1}^{a, b, r}\right)=\operatorname{gcd}(a, b)$ for non-negative integer $n$. Then we have

$$
\begin{aligned}
\operatorname{gcd}\left(U_{n+1}^{a, b, r}, U_{n+2}^{a, b, r}\right) & =\operatorname{gcd}\left(U_{n+1}^{a, b, r}, r U_{n+1}^{a, b, r}+U_{n}^{a, b, r}\right) \\
& =\operatorname{gcd}\left(U_{n+1}^{a, b, r}, U_{n}^{a, b, r}\right) \\
& =\operatorname{gcd}(a, b) .
\end{aligned}
$$

This completes the proof of Theorem 4 . 
Let $r=1,2$ in Theorem 4 . We can get the corollary.

Corollary 5. For every non-zero integers $a, b$ and non-negative integer $n$, we have

(1) $\operatorname{gcd}\left(G_{n}^{a, b}, G_{n+1}^{a, b}\right)=\operatorname{gcd}(a, b)$,

(2) $\operatorname{gcd}\left(P_{n}^{a, b}, P_{n+1}^{a, b}\right)=\operatorname{gcd}(a, b)$.

Corollary 6. For non-negative integer n, we have

(1) $\operatorname{gcd}\left(F_{n}, F_{n+1}\right)=1$,

(2) $\operatorname{gcd}\left(L_{n}, L_{n+1}\right)=1$,

(3) $\operatorname{gcd}\left(P_{n}, P_{n+1}\right)=1$,

(4) $\operatorname{gcd}\left(Q_{n}, Q_{n+1}\right)=2$.

Theorem 7. For every non-zero integer a and non-negative integers $n, m$, we have

$$
\operatorname{gcd}\left(G_{n}^{a, a}, G_{m}^{a, a}\right)=|a| F_{\operatorname{gcd}(m, n)}
$$

Proof.

$$
\begin{aligned}
\operatorname{gcd}\left(G_{m}^{a, a}, G_{n}^{a, a}\right) & =\operatorname{gcd}\left(a F_{m-2}+a F_{m-1}, a F_{n-2}+a F_{n-1}\right) \\
& =|a| \cdot \operatorname{gcd}\left(F_{m-2}+F_{m-1}, F_{n-2}+F_{n-1}\right) \\
& =|a| \cdot \operatorname{gcd}\left(F_{m}, F_{n}\right) \\
& =|a| F_{\operatorname{gcd}(m, n)} .
\end{aligned}
$$

Lemma 8. For every non-zero integers $a, b, r$ and non-negative integer $n$, we have

$$
\operatorname{gcd}\left(G_{n}^{a, b}, G_{n}^{a, b+r}\right)=\operatorname{gcd}\left(a F_{n-2}+b F_{n-1}, r F_{n-1}\right) .
$$

Proof.

$$
\begin{aligned}
\operatorname{gcd}\left(G_{n}^{a, b}, G_{n}^{a, b+r}\right) & =\operatorname{gcd}\left(a F_{n-2}+b F_{n-1}, a F_{n-2}+(b+r) F_{n-1}\right) \\
& =\operatorname{gcd}\left(a F_{n-2}+b F_{n-1}, a F_{n-2}+b F_{n-1}+r F_{n-1}\right) \\
& =\operatorname{gcd}\left(a F_{n-2}+b F_{n-1}, r F_{n-1}\right) .
\end{aligned}
$$

Corollary 9. For every non-zero integers $a, b, r$ and non-negative integer $n$, we have

(1) $\operatorname{gcd}\left(G_{n}^{a, b}, G_{n}^{a, b+1}\right)=\operatorname{gcd}\left(a, F_{n-1}\right)$,

(2) if $r \mid b$, then $\operatorname{gcd}\left(G_{n}^{a, b}, G_{n}^{a, b+r}\right)=\operatorname{gcd}\left(a F_{n-2}, r F_{n-1}\right)$. 
Theorem 10. For every positive integer $n$, then $\operatorname{gcd}\left(F_{n}, L_{n}\right)$ is 1 or 2 . Moreover, we have

$$
\operatorname{gcd}\left(F_{n}, L_{n}\right)=2 \text { if and only if } 3 \mid n \text {. }
$$

Proof. We set $a=b=1$ and $r=2$ in Lemma 8, then $\operatorname{gcd}\left(F_{n}, L_{n}\right)=\operatorname{gcd}\left(G_{n}^{1,1}, G_{n}^{1,3}\right)=$ $\operatorname{gcd}\left(F_{n-2}+F_{n-1}, 2 F_{n-1}\right)=\operatorname{gcd}\left(F_{n}, 2 F_{n-1}\right)$. Since $\operatorname{gcd}\left(F_{n}, F_{n+1}\right)=1$, we have $\operatorname{gcd}\left(F_{n}, L_{n}\right)$ is 1 or 2 . By Theorem 2, it is clear that $\operatorname{gcd}\left(F_{n}, L_{n}\right)=2$ if and only if $3 \mid n$.

Table 1: $\operatorname{gcd}\left(F_{n}, L_{n}\right)$ for some values of $n$.

\begin{tabular}{|c|c|c|c|c|c|c|c|c|c|c|c|}
\hline$n$ & 0 & 1 & 2 & 3 & 4 & 5 & 6 & 7 & 8 & 9 & 10 \\
\hline$F_{n}$ & 0 & 1 & 1 & 2 & 3 & 5 & 8 & 13 & 21 & 34 & 55 \\
\hline$L_{n}$ & 2 & 1 & 3 & 4 & 7 & 11 & 18 & 29 & 47 & 76 & 123 \\
\hline $\operatorname{gcd}\left(F_{n}, L_{n}\right)$ & 2 & 1 & 1 & 2 & 1 & 1 & 2 & 1 & 1 & 2 & 1 \\
\hline
\end{tabular}

Theorem 11. For every non-zero integers $a, b, r$ and non-negative integer $n$, we have

(1) $\operatorname{gcd}\left(U_{2 n}^{a, b, r}, U_{2 n+2}^{a, b, r}\right)=\operatorname{gcd}(r a, b)$,

(2) $\operatorname{gcd}\left(U_{2 n+1)}^{a, b, r}, U_{2 n+3}^{a, b, r}\right)=\operatorname{gcd}(a, r b)$.

Proof. We will proceed by induction on $n$. It is clear that $\operatorname{gcd}\left(U_{0}^{a, b, r}, U_{2}^{a, b, r}\right)=\operatorname{gcd}(b-r a, b)=$ $\operatorname{gcd}(r a, b)$ and $\operatorname{gcd}\left(U_{1}^{a, b, r}, U_{3}^{a, b, r}\right)=\operatorname{gcd}(a, r b+a)=\operatorname{gcd}(a, r b)$.

First, we assume that $\operatorname{gcd}\left(U_{2 n}^{a, b, r}, U_{2 n+2}^{a, b, r}\right)=\operatorname{gcd}(r a, b)$. Then we have

$$
\begin{aligned}
\operatorname{gcd}\left(U_{2 n+2}^{a, b, r}, U_{2 n+4}^{a, b, r}\right) & =\operatorname{gcd}\left(U_{2 n+2}^{a, b, r}, r U_{2 n+3}^{a, b, r}+U_{2 n+2}^{a, b, r}\right) \\
& =\operatorname{gcd}\left(U_{2 n+2}^{a, b, r}, r U_{2 n+3}^{a, b, r}\right) \\
& =\operatorname{gcd}\left(U_{2 n+2}^{a, b, r}, r\left(r U_{2 n+2}^{a, b, r}+U_{2 n+1}^{a, b, r}\right)\right) \\
& =\operatorname{gcd}\left(U_{2 n+2}^{a, b, r}, r U_{2 n+1}^{a, b, r}\right) \\
& =\operatorname{gcd}\left(U_{2 n+2}^{a, b, r}, U_{2 n+2}^{a, b, r}-U_{2 n}^{a, b, r}\right) \\
& =\operatorname{gcd}\left(U_{2 n+2}^{a, b, r}, U_{2 n}^{a, b, r}\right) \\
& =\operatorname{gcd}(r a, b) .
\end{aligned}
$$

Finally, we assume that $\operatorname{gcd}\left(U_{2 n+1}^{a, b, r}, U_{2 n+3}^{a, b, r}\right)=\operatorname{gcd}(a, r b)$. Then we have

$$
\begin{aligned}
\operatorname{gcd}\left(U_{2 n+3}^{a, b, r}, U_{2 n+5}^{a, b, r}\right) & =\operatorname{gcd}\left(U_{2 n+3}^{a, b, r}, r U_{2 n+4}^{a, b, r}+U_{2 n+3}^{a, b, r}\right) \\
& =\operatorname{gcd}\left(U_{2 n+3}^{a, b, r}, r U_{2 n+4}^{a, b, r}\right) \\
& =\operatorname{gcd}\left(U_{2 n+3}^{a, b, r}, r\left(r U_{2 n+3}^{a, b, r}+U_{2 n+2}^{a, b, r}\right)\right) \\
& =\operatorname{gcd}\left(U_{2 n+3}^{a, b, r}, r U_{2 n+2}^{a, b, r}\right) \\
& =\operatorname{gcd}\left(U_{2 n+3}^{a, b, r}, U_{2 n+3}^{a, b, r}-U_{2 n+1}^{a, b, r}\right) \\
& =\operatorname{gcd}\left(U_{2 n+3}^{a, b, r}, U_{2 n+1}^{a, b, r}\right) \\
& =\operatorname{gcd}(a, r b) .
\end{aligned}
$$

This completes the proof of Theorem 11. 
Let $r=1,2$ in Theorem 11 . We can get the corollary.

Corollary 12. For every non-zero integers $a, b$ and non-negative integer $n$, we have

(1) $\operatorname{gcd}\left(G_{n}^{a, b}, G_{n+2}^{a, b}\right)=\operatorname{gcd}(a, b)$,

(2) $\operatorname{gcd}\left(P_{n}^{a, b}, P_{n+2}^{a, b}\right)= \begin{cases}\operatorname{gcd}(2 a, b), & \text { if } n \text { is even } \\ \operatorname{gcd}(a, 2 b), & \text { if } n \text { is odd }\end{cases}$

Corollary 13. For non-negative integer $n$, we have

(1) $\operatorname{gcd}\left(F_{n}, F_{n+2}\right)=1$,

(2) $\operatorname{gcd}\left(L_{n}, L_{n+2}\right)=1$,

(3) $\operatorname{gcd}\left(P_{n}, P_{n+2}\right)= \begin{cases}2, & \text { if } n \text { is even } \\ 1, & \text { if } n \text { is odd }\end{cases}$

(4) $\operatorname{gcd}\left(Q_{n}, Q_{n+2}\right)=2$.

Theorem 14. For every integers $a, b$ and non-negative integer $n$, we have

$$
P_{n+2}^{a, b}=a P_{n}+b P_{n+1} .
$$

Proof. We will proceed by induction. We will proceed by induction on $n$. We have $P_{2}^{a, b}=b=$ $a P_{0}+b P_{1}$ and $P_{3}^{a, b}=a+2 b=a P_{1}+b P_{2}$. Now, we assume that $P_{k+2}^{a, b}=a P_{k}+b P_{k+1}$ for every $k \in\{0,1,2, \ldots, n\}$. Then

$$
\begin{aligned}
P_{n+3}^{a, b} & =2 P_{n+2}^{a, b}+P_{n+1}^{a, b} \\
& =2\left(a P_{n}+b P_{n+1}\right)+\left(a P_{n-1}+b P_{n}\right) \\
& =a\left(2 P_{n}+P_{n-1}\right)+b\left(2 P_{n+1}+P_{n}\right) \\
& =a P_{n+1}+b P_{n+2} .
\end{aligned}
$$

This completes the proof of Theorem 14 .

Lemma 15. For every non-zero integers $a, b, r, s$ and non-negative integer $n$, we have

$$
\operatorname{gcd}\left(P_{n}^{a, b}, P_{n}^{a+r, b+s}\right)=\operatorname{gcd}\left(a P_{n-2}+b P_{n-1}, r P_{n-2}+s P_{n-1}\right) .
$$

Proof.

$$
\begin{aligned}
\operatorname{gcd}\left(P_{n}^{a, b}, P_{n}^{a+r, b+s}\right) & =\operatorname{gcd}\left(a P_{n-2}+b P_{n-1},(a+r) P_{n-2}+(b+s) P_{n-1}\right) \\
& =\operatorname{gcd}\left(a P_{n-2}+b P_{n-1}, a P_{n-2}+b P_{n-1}+r P_{n-2}+s P_{n-1}\right) \\
& =\operatorname{gcd}\left(a P_{n-2}+b P_{n-1}, r P_{n-2}+s P_{n-1}\right) .
\end{aligned}
$$

Theorem 16. For every non-negative integer $n$, then $\operatorname{gcd}\left(P_{n}, Q_{n}\right)$ is 1 or 2 . Moreover, we have $\operatorname{gcd}\left(P_{n}, Q_{n}\right)=2$ if and only if $n$ is even.

Proof. We set $a=r=1, b=2$ and $s=4$ in Lemma 15, then

$$
\operatorname{gcd}\left(P_{n}, Q_{n}\right)=\operatorname{gcd}\left(P_{n}^{1,2}, P_{n}^{2,6}\right)=\operatorname{gcd}\left(P_{n-2}+2 P_{n-1}, P_{n-2}+4 P_{n-1}\right)=\operatorname{gcd}\left(P_{n}, 2 P_{n-1}\right) .
$$

Since $\operatorname{gcd}\left(P_{n}, P_{n+1}\right)=1$, we have $\operatorname{gcd}\left(P_{n}, Q_{n}\right)$ is 1 or 2 . By Theorem 2, it is clear that $\operatorname{gcd}\left(P_{n}, Q_{n}\right)=2$ if and only if $n$ is even. 
Table 2: $\operatorname{gcd}\left(P_{n}, Q_{n}\right)$ for some values of $n$.

\begin{tabular}{|c|c|c|c|c|c|c|c|c|c|c|c|}
\hline$n$ & 0 & 1 & 2 & 3 & 4 & 5 & 6 & 7 & 8 & 9 & 10 \\
\hline$P_{n}$ & 0 & 1 & 2 & 5 & 12 & 29 & 70 & 169 & 408 & 985 & 2,378 \\
\hline$Q_{n}$ & 2 & 2 & 6 & 14 & 34 & 82 & 198 & 478 & 1,154 & 2,786 & 6,726 \\
\hline $\operatorname{gcd}\left(P_{n}, Q_{n}\right)$ & 2 & 1 & 2 & 1 & 2 & 1 & 2 & 1 & 2 & 1 & 2 \\
\hline
\end{tabular}

\section{Acknowledgements}

The author would like to thank the referee for his/her valuable comments. The author would like to thank the Ubon Ratchathani Rajabhat University for the full support in this research.

\section{References}

[1] Adb-Elhameed, W. M., \& Zeyada, N. A. (2015) Some new identities of generalized Fibonacci and generalized Pell numbers via a new type of numbers, preprint, http: //arxiv.org/abs/1511.07588v1.

[2] Chen, K. W. (2011) Greatest common divisors in shifted Fibonacci sequences. J. Integer Sequences, 14, 1-8 (Article 11.4.7). Available online at: https://cs . uwaterloo. ca/journals/JIS/VOL14/Chen/chen70.pdf.

[3] Dudley, U., \& Tucker, B. (1971) Greatest common divisors in altered Fibonacci sequences, The Fibonacci Quarterly, 9, 89-91.

[4] Koshy, T. (2014) Pell and Pell-Lucas Numbers with Applications, Springer, Berlin.

[5] Koshy, T. (2001) Fibonacci and Lucas Numbers with Applications, Wiley-Interscience Publications.

[6] McDaniel, W. L. (1991) The G.C.D. in Lucas Sequences and Lehmer Number Sequences, The Fibonacci Quarterly, 29, 24-29.

[7] Raji, W. (2004) An introductory course in elementary number theory, Mobius. 